\title{
An Analog Processor Array Implementing Interconnect-Efficient Reference Data Shift and SAD/SSD Extraction for Motion Estimation
}

\author{
Jonne Poikonen, ${ }^{1}$ Mika Laiho, ${ }^{1}$ Ari Paasio, ${ }^{1}$ Lauri Koskinen, ${ }^{2}$ and Kari Halonen ${ }^{2}$ \\ ${ }^{1}$ Department of Information Technology, University of Turku, 20014 Turku, Finland \\ ${ }^{2}$ Electronic Circuit Design Laboratory, Helsinki University of Technology, P.O. Box 300, 02015 Espoo, Finland
}

Correspondence should be addressed to Jonne Poikonen, jokapo@utu.fi

Received 25 September 2008; Accepted 30 January 2009

Recommended by Diego Cabello Ferrer

A cellular analog processor array for use in variable block-size motion estimation with a new simple method for shifting reference image data is presented. The new shift method leads to a greatly reduced number of neighborhood connections for each cell of the array, and allows for all shifts within the $[8,8]$ search area to be performed in a single step, with simple digital controls. The new shift circuitry, together with some other cell and system level optimizations, reduces silicon area and array layout complexity, enabling faster and more efficient parallel full search motion estimation hardware. A $32 \times 32$ cell parallel analog test array for reference-shift with a maximum block-size of $16 \times 16$, as well as absolute value/quadratic processing for variable block-size analog motion estimation (AME) has been designed in a $0.13 \mu \mathrm{m}$ CMOS technology.

Copyright (C) 2009 Jonne Poikonen et al. This is an open access article distributed under the Creative Commons Attribution License, which permits unrestricted use, distribution, and reproduction in any medium, provided the original work is properly cited.

\section{Introduction}

Cameras with (multi-)megapixel sensors have become ubiquitous in even relatively low-end mobile phones. While this makes good quality still imaging possible, the limited amount of memory and processing power in such a batterypowered mobile platform often prohibits the use of the best available image quality for capturing video streams; typically a considerably poorer video capture resolution is used. The strong overall trend of memory technology scaling enables the integration of increasing amounts of memory within mobile phones. However, the increase of processing power which is required for real-time processing of the video stream is considerable.

An integral part of all video standards is motion estimation (ME), which can take up to $80 \%$ of the power consumption of a video encoder. For small frame sizes, the ME power consumption can be reduced through algorithmic methods, however, for megapixel resolutions these solutions are not sufficient. Without new optimized circuit techniques, the power consumption due to the motion estimation process will grow beyond the capabilities of small batterypowered platforms.

The currently applied video standards for mobile terminals (e.g., H.264) employ Block-Based Motion Estimation (BBME), and preferably variable block-size motion estimation. The most fundamental operation required for BBME is the shift of the reference-block data, to which the current frame data is compared, after which the bestmatching new block position is determined with relatively simple processing. A performance advantage has been sought from performing the motion estimation operation in the analog domain and by employing a CNN-type [1] parallel processor array [2-8].

This paper describes the implementation of parallel processing hardware for an analog motion estimation (AME) array, with a focus on the implementation of a new reference data shift method. The proposed shift implementation leads to a significant reduction in the required cell interconnections, enabling a $[8,8]$ cell search range to be implemented with a simpler array-level wiring than in previous implementations and with simple controllability. 


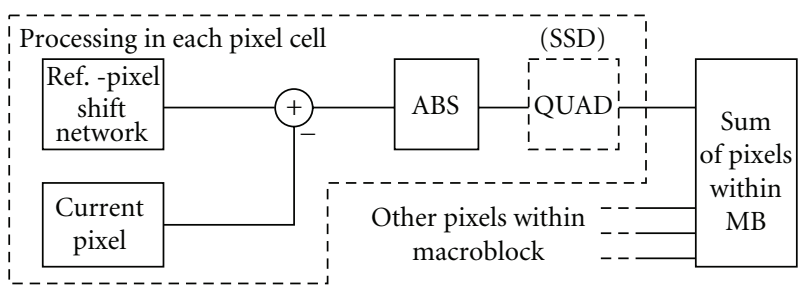

FIGURE 1: Cell-level functionality required for BBME.

This paper extends the original paper proposing the new reference-shift method [9], by also describing in detail the implementation of other circuitry for the array cell as well as presenting the implementation of a $32 \times 32$ cell AME test chip that has been designed and submitted for manufacturing.

The paper is divided into seven Sections. Section 2 discusses some implementation issues relating to a motion estimation array realization, Section 3 describes the new reference shift method in detail, and Section 4 examines the other circuitry in the array cell. In section 5 some important implementation issues are discussed, Section 6 describes the designed test array and examines the performance of other proposed motion estimation processors, and finally some conclusions are drawn in Section 7.

\section{Analog Motion Estimation Array}

Variable block-size motion estimation is based on comparing a macroblock of pixels, typically from $4 \times 4$ to $16 \times 16$ pixels, in the current image frame (C-frame) to blocks of the same size within the search area of a reference frame (R-frame). The position where the best matching of the macroblocks in the different frames is achieved represents the estimate for the motion in the image, that is, the motion vector. The matching at each position is evaluated by using a matching criterion which is typically either the Sum of Absolute Differences (SAD) or the Sum of Squared Differences (SSD) between the individual macroblock pixel values in the current and reference frames. The optimal selection of the method depends on the type of hardware implementation, SAD is more typically used in digital implementations because the required calculations are much simpler. A fair approximation of SSD can be easily implemented with current-mode analog circuitry, however, the accuracy compared to an actual squaring operation is limited by the nonideal characteristics of transistors, especially in modern deep-submicron technologies and with low power supply voltages. Figure 1 demonstrates the cell operations required for an analog motion estimation array. The different circuit blocks will be discussed in detail in the following chapters.

In principle, the optimal implementation of analog motion estimation would be to integrate the motion estimation circuitry together with each pixel in the photosensor array. By not having to convert the analog pixel values into digital form before motion estimation, considerable power savings could be achieved and the processing could be performed for the whole frame in a fully parallel manner. In reality this is not feasible for a megapixel sensor array, due to the resulting excessive silicon area required by the processing circuitry per pixel. Also, without A/D conversion, the input frames would have to be stored in analog memories, which creates many implementation and performance difficulties, especially with advanced CMOS technology.

Because of these reasons, a more realistic alternative is to separate the imager array and the analog motion estimation processor. Even in this case the processor array cannot be practically designed with the same spatial resolution as a very large sensor. There are different ways to overcome this problem. The processor array can, that is, be implemented with the same number of columns than the image sensor, however, with only a limited number of rows. Another possibility is to implement the processor as a significantly smaller but symmetrical array, which is applied to the larger image frame in a windowed manner. Making a single processing cell as simple and small as possible is still crucial, since it enables the implementation of a larger processing window, reducing the number of required iterations for a large image size, and thus increasing the possible processing speed.

The actual motion estimation process performed by the array processor should be fast enough not to limit the achievable frame rate or frame size. The efficiency of the implementation is also heavily dependent on the speed of data transfer between the imager and the motion estimation processor, which means that the communication scheme should be carefully designed. The first requirement can be fairly easily achieved by using efficient analog current-mode signal processing. Because the analog image data from a sensor is always converted into digital form for further handling and storage, also the data communication with an external motion estimation core should be digital. This enables high-speed I/O operations and makes a separate analog motion estimation processor compatible with a system environment which is otherwise fully digital.

In a motion estimation processor with digital input, each cell of the AME array has to include two (typically 8-bit) digital to analog converters for providing data for the two frames to be compared, and the corresponding in-cell digital memory elements. The digital I/O for the processor is heavily asymmetrical; the only output required from the AME processor is digital motion vector data, that is, the identification of the shift location which results in the smallest block difference. The actual image data does not have to be read out of the processor array. The motion estimation circuitry does not have to have, nor should have, any direct effect on the image data itself. This will prevent additional image errors due to inevitable inaccuracies in analog operation.

\section{Shifting of Reference Data}

In principle, the switching operation could be performed by moving the pixel values step-by-step through only first neighborhoods connections. However, this would require current memories for intermediate storage, if implemented 


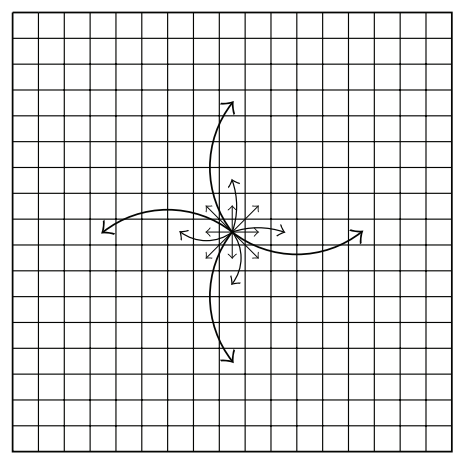

FIgURE 2: Cell neighborhood connections. Because the connections are bidirectional, the number of actual wires per cell is only 8 .

in an analog fashion. The large number of sequential current memory read/write operations may slow down the shift operation and cause additional inaccuracy, and can potentially lead to higher power consumption from increased control signal activity. The proposed shift method also allows the efficient use of possible optimized search patterns, in addition to an exhaustive full search. Shifting the values cellby-cell would make this much more inefficient.

The shift operation in a massively parallel array could also be performed in a fully digital manner, solving the problems of interconnect complexity and analog inaccuracies. On the other hand, this may lead to many new design challenges, that is, in terms of circuit complexity, power consumption and the implementation of the actual in-cell processing. However, the prospect is a very interesting direction for future research.

Figure 2 shows the neighborhood connections available in the network. Each connection between cells operates bidirectionally and is shared between two cells; the actual number of physical wires per cell is only half of the number of direct neighborhood connections. The choice between input and output operations for each direction is implemented with switches and logic inside the cell.

Because neighborhood connections to the 2nd and 5th neighbors are only available in the cardinal directions $(\mathrm{N}$, $\mathrm{E}, \mathrm{S}$, and $\mathrm{W}$ ), the shifts to the diagonal directions are implemented by using the same neighborhood twice in the same shift operation. The principle of the double shift is that first a connection to either $\mathrm{N}$ or $\mathrm{S}$ is used, after which the input to the cell is fed directly to the $\mathrm{E}$ or $\mathrm{W}$ connection of the same neighborhood. After this, the signal can either be taken into the target cell or to a lower neighborhood, from 5th to 2 nd or from 2nd to the 1st neighborhood. By combining effectively 8 -connected 5 th and 2 nd neighborhoods with an actually 8-connected local neighborhood, all cells within an 8 -cell search area can be accessed (from 1 to $5+2+1$ ). Because the output directions in the different neighborhoods can be controlled individually, that is, a shift with a length of 4 can be implemented simply by moving into the opposite direction in the lower neighborhood: $\operatorname{East}(4)=$ East(5) West(1). Figure 3 shows two examples of shift operations with the proposed connectivity.

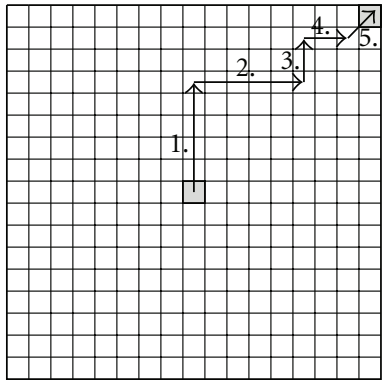

(a)

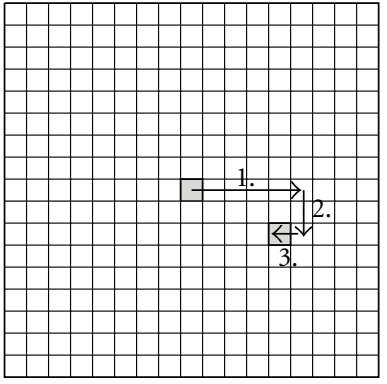

(b)
Figure 3: (a) Cell neighborhood connections. Example of (b) $[8,8]$ shift, (c) [4,-2] shift.

The approach proposed here significantly simplifies the connectivity in the array, compared to the previously proposed methods $[5,6]$. The number of neighborhood connections per cell is now only 16, as opposed to 30 [5]. Although the number of separate cell connections required for some shifts is now 5 compared to a previous maximum of 3, all shifts are still implemented directly in one step, without having to store any pixel values in intermediate cells. The hierarchically implemented shift procedure is very straightforward and simple to control, requiring roughly 20 global control signals, which could be reduced by including in-cell control signal decoding. All controls could also be generated in-cell with a dedicated state-machine, however, in that case the cell complexity and area would be greatly increased. The metal pitch in current CMOS technologies is very small, which means that the number of global wires required for the proposed circuitry can be easily routed even over a fairly small cell size.

The layout design complexity is also greatly reduced with the proposed shift network, because of the fewer intercell connections and a fully symmetrical wiring arrangement; in [5] the connections were asymmetrical, which makes the layout design very complicated. In this case, since all connections are bidirectional, the number of individual neighborhood wires that have to be implemented for each cell is only 8 and the rest of the connections are realized automatically through symmetry.

3.1. Shift Configuration. The switch configuration for a single cell, used for the shift operation, is shown in Figure 4. The local input to the cell is provided by a current-mode Currentframe DAC (C-DAC) whereas the Reference-frame DAC (RDAC) provides the output value of the cell which is shifted through the network. The local C-DAC current value is subtracted from the shifted R-DAC output, propagated from the source cell of the shift, and the current difference is applied to the ABS + QUAD block, which is implemented with very simple analog current-mode circuitry.

During the shift operation, the output current of the R-DAC is lead directly through a series of simple NMOStransistor switches to the target cell. The simplified control signal configuration for the shift is as follows. 


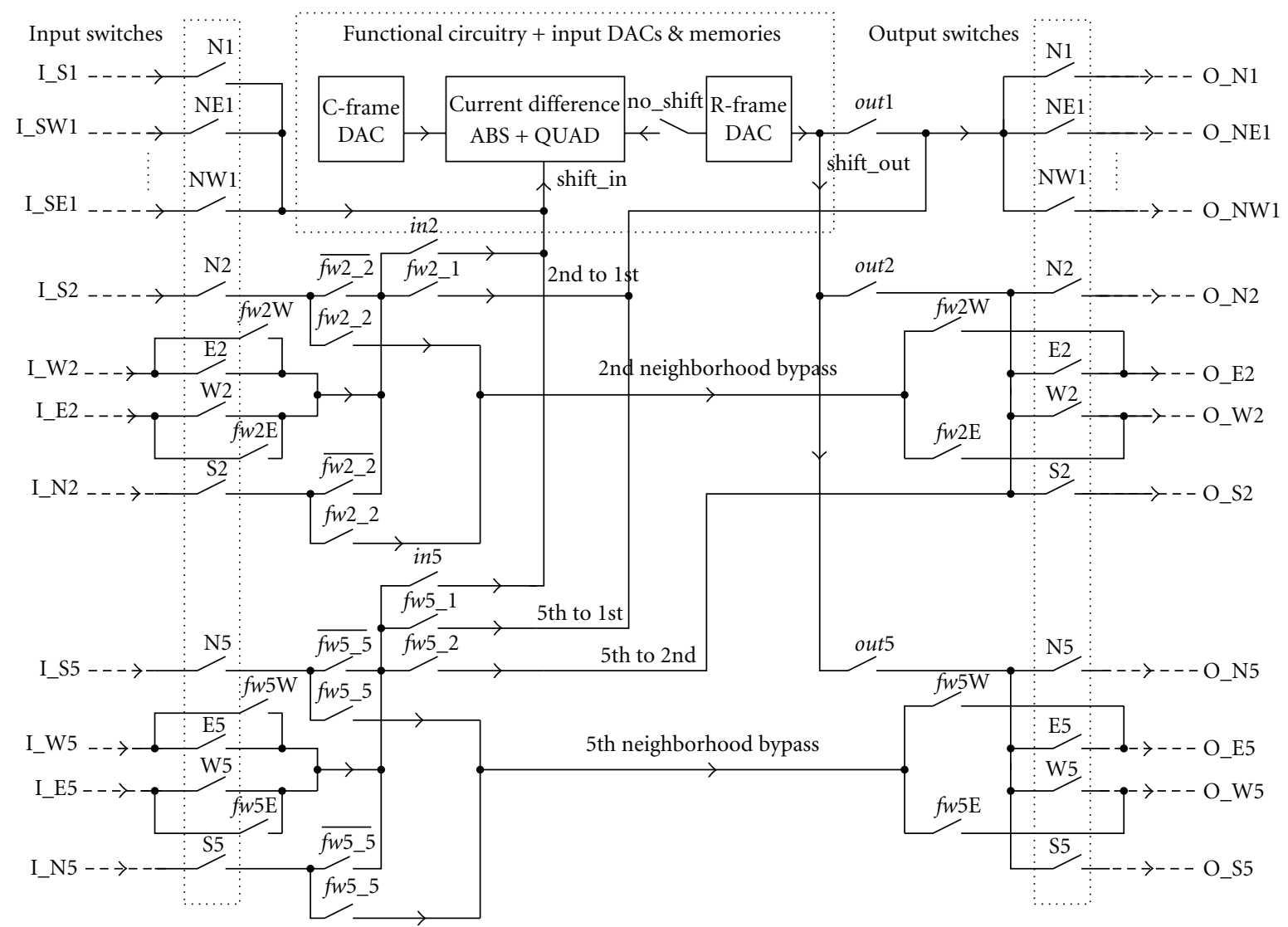

FIGURE 4: Cell switch configuration for the reference shift. All switches are implemented with NMOS transistors.

3.1.1. Selection of the Correct Output Neighborhood and Direction. Global control signals out 1 , out2, and out5 are used to select the neighborhood to which the R-DAC output current is propagated. The direction controls are implemented with 3 bits for the first neighborhood and with 2 bits each for the 2nd and 5th neighborhoods; a single output/input switch in the simplified schematic of Figure 4 is actually implemented either as 3 or 2 NMOS transistor switches in series. The control signal noshift is used to implement a $[0,0]$ shift.

3.1.2. Selection of Propagation to a Lower Neighborhood. Global signals $f w 5 \_1, f w 5 \_2$, and $f w 2 \_1$ are used for moving hierarchically to a lower (closer) cell neighborhood, in order to implement all necessary propagation paths. From the 5th neighborhood the signal can be propagated either to the 2 nd or 1 st neighborhoods and the 8 -connected 1 st neighborhood can be reached from the 2 nd neighborhood.

\subsubsection{Selection of the Direction of Secondary Propagation.} In the 2 nd or 5 th neighborhoods, two propagation directions can be used at the same time. When the signal is propagated either to North or South, another wire in the same neighborhood can be used, either to East or West. The local control signals $f w 2 \_2$ and $f w 5 \_5$ in the cell are implemented as $O R\left(f w x_{-} E, f w x_{-} W\right)$. This means that if neither secondary direction (E/W) is globally enabled, the secondary connection will not be used (e.g., $f w 2 \_2=$ $L O W$ ), and the first 2nd or 5th neighborhood connection (N/S) has to be directed either to the input of the target cell or to a lower neighborhood.

3.1.4. Selection of the Input Neighborhood. The neighborhood which provides the input to the target cell is selected with the global signals in 2 and in5. Input switches are not required for the 1st neighborhood, because if a signal is applied to any 1st neighborhood output wire, it is always taken directly to the input of the neighboring target cell; propagation to an upper hierarchy level is not possible. Separate input and output direction switches are still required because the cell interconnect wires are used bidirectionally. The controls for the input direction switches are hardwired opposite to the output switches, so that each neighborhood wire can only be accessed by a cell in one direction at a time.

3.2. Shift Network Complexity. The cell circuitry required for the shifting consists of approximately 130 transistors, of which roughly 100 are NMOS-type switches, while the others account for additional inverters and logic within the cell. The complexity of the cell circuitry is reduced, for example, by implementing the shift-direction decoding directly with the switches used for the shift operation itself, 
instead of using separate decoder circuitry. The realized shift circuitry is rather compact, however in future research and implementations some additional optimization may still be possible.

The complexity of the cell circuitry could be further reduced by separating the output and input wires used for the shift. If each neighborhood connection wire was made one-directional (input/output), input direction selection would not be required and a part of the switches could be omitted. This would reduce circuit area and the resistive effects discussed later, however, the neighborhood wiring complexity would be greatly increased because the number of physical wires would be doubled. In this case, simple interconnect wiring was targeted. Also, the area requirements of additional wiring may counteract some of the area savings from a reduced number of transistors.

A compromise between the number of switches and interconnect layout complexity could be reached by implementing only the first neighborhood connections with separate input/output wiring. This would reduce the number of transistors but would not require doubling the number of long interconnects, which have to pass over other cells, thus limiting the additional layout design more complexity.

\section{Other Cell Circuitry}

In addition to the shift network, each cell of the array includes the C-frame and R-frame DACs, which are NMOStype 8-bit current mode binary-weighted converters, 16 static digital memory elements for storing the DAC input codes and the actual analog processing circuitry. The processing circuitry consists of a current-mode absolute value circuit followed by a current squarer circuit. This processing circuitry is effectively the same as in an earlier proposed AME designs $[7,8]$, however, the cell circuitry has been optimized for the new array design, which does not include current memories and in-cell current averaging. After the fairly simple in-cell analog processing, the summing of the cell outputs, within variable-sized macroblocks, has to be performed, and the sums for different macroblock locations have to be compared to find the best matching shift vector. In the current test chip design this is done with separate processing outside the chip.

4.1. Reference Source DAC Swapping. During the motion estimation procedure for a continuous stream of frames, after the motion vector for a frame has been determined, the reference frame will typically become the current frame for the next motion estimation step. In the AME array, where the input images are provided by in-cell DACs, the input data for the next C-frame is already stored in the cell as the R-frame for the previous operation. It is therefore desirable to use that frame data instead of writing both $\mathrm{C}$-frame and R-frame data into each cell of the array for every frame of the image stream.

Because both the C-frame and the R-frame are stored in static digital memory registers inside the cell, the R-frame register could be simply written into the $\mathrm{C}$-frame register. However, it is easier and more power-efficient to simply

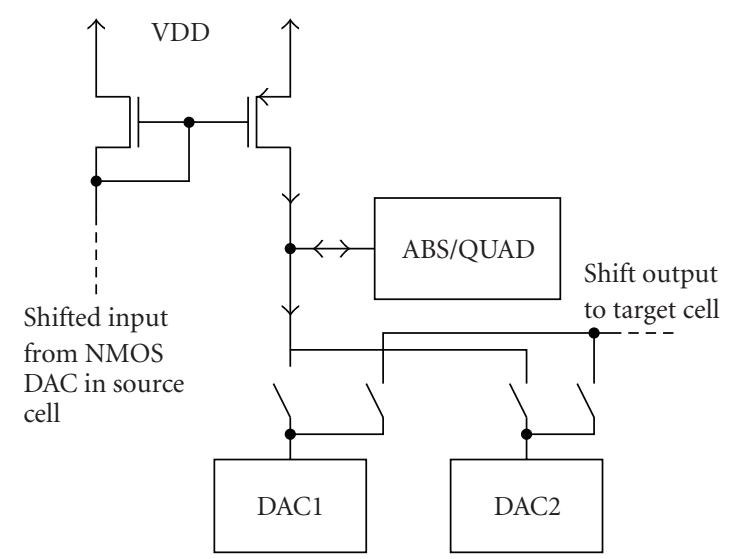

FIGURE 5: Input configuration for current shift with DAC swapping.

swap the outputs of the two in-cell DACs in every successive motion estimation step. Because current-output DACs and current-mode processing are used, the output current of a DAC can be simply redirected through a switch either to the local difference block (used as C-DAC) or to the shift network (used as R-DAC). This way only the reference frame data has to be written into the cells in each motion estimation step, and power and time is saved during the read-in phase of the processor array.

The benefit of the DAC swapping is only truly realized if a full image-sized processor array is implemented, that is, the whole image is processed at the same time. However, it maybe also be somewhat useful for $\mathrm{I} / \mathrm{O}$ optimization in a windowed operation with a relatively large window (array) size, compared to the complete image, so that the swapping can be used for the last processed window of the image, to begin a new sweep with the existing DAC data. The swapping of the DACs and the cell input configuration are illustrated in Figure 5.

4.2. Absolute Value and Quadrature Operation. Figure 6 shows the actual processing circuitry in each cell of the array, along with the transistor sizes. The circuitry consists of a current-mode absolute value circuit and a current squarer. Some additional switches have been added to make the cell operation more flexible, so that either absolute value or quadrature output can be used for the cells.

The difference between the shifted reference frame pixel value and the local current-frame pixel value is realized at the input of the absolute value (ABS) block as a simple current subtraction. The absolute value circuit is implemented as proposed in [10]. Depending on whether the input current to the circuit is positive or negative (towards or away from the input node), the input voltage will be driven either higher or lower. The input voltage swing is amplified by the inverter, which is connected between the input node and the gates of the NMOS and PMOS transistors at the input of the ABSclock. The inverter helps to efficiently close the unwanted and open the correct current path (direction) through the rectifier. This results in reduced voltage variation at the input node of the rectifier and thus improved performance, 


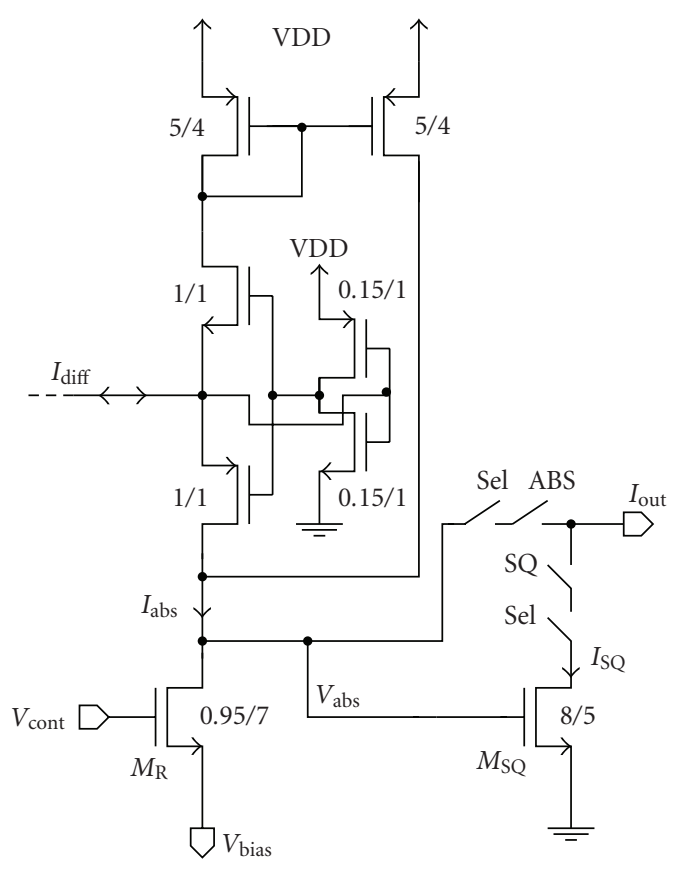

Figure 6: Absolute value and quadrature circuitry. The applied transistor dimensions $(W / L)$ are in micrometers.

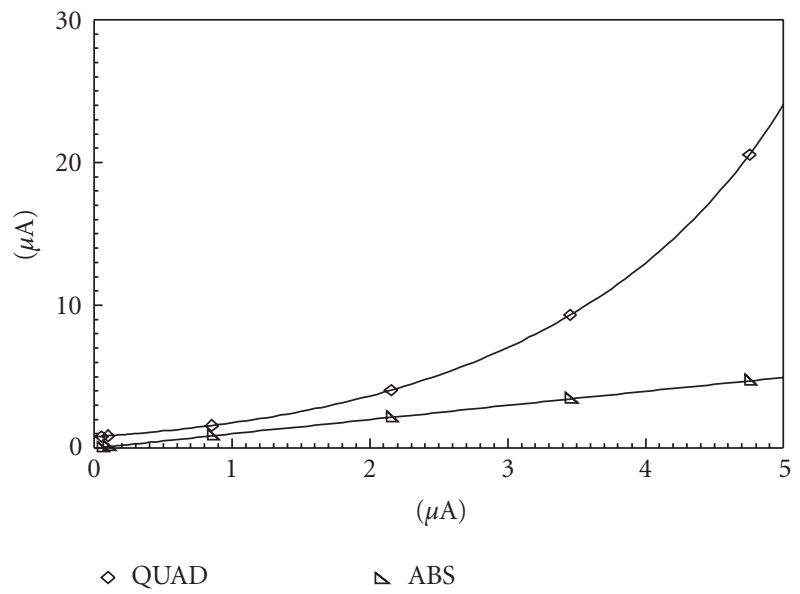

Figure 7: Simulated absolute value and quadratic responses of the cell.

compared to simpler rectifier circuits [11]. The addition of the inverter adds some extra complexity and power consumption, however, in this case the overall circuitry is so simple that the performance advantage is more significant.

When the current to the absolute value circuit is zero, the input voltage is somewhere in the middle of the power rails and a race situation is created in the inverter, leading to static current consumption. The magnitude of this current is limited in the cell to $<1 \mu \mathrm{A}$ by making the inverter transistors long and very narrow as can be seen from Figure 6 .

The output of the absolute value circuit is taken to an NMOS transistor $M_{\mathrm{R}}$ which is effectively operating as a resistor. In normal operation, the gate voltage of transistor $M_{\mathrm{R}}$ is set to VDD and the voltage over $M_{\mathrm{R}}$ is relatively small.
Therefore, the transistor is operating in the triode region as an approximately linear resistor. The source bias voltage of $M_{\mathrm{R}}$ can be adjusted in order to set the correct input range for the subsequent squaring transistor $M_{\mathrm{SQ}}$.

The squaring transistor $M_{\mathrm{SQ}}$ takes as its input the approximately linear output voltage $V_{\text {abs }}$. The transistor is biased so that it is operating in saturation and thus provides an output current which is approximately quadratic with respect to the input voltage:

$$
I_{\mathrm{SQ}} \approx \frac{\beta}{2}\left(V_{\mathrm{abs}}-V_{\mathrm{TN}}\right)^{2} .
$$

It has to be noted that the squaring is only approximate and the accuracy is also affected by the inevitable nonlinearity of the ABS output. Also, the transistor $M_{\mathrm{SQ}}$ is, for layout reasons, actually implemented as two $W / L=8 / 2.5 \mu \mathrm{m}$ NMOS devices in series. This has only a very small effect on the quadratic output response of the cell, as opposed to using a single transistor. It has been shown that an exact quadrature operation is not really necessary, nor even the most optimal solution [12]. Figure 7 shows the simulated responses of the absolute value circuit and the quadrature transistor when the input current was swept from 0 to $5 \mu \mathrm{A}$, which can be considered to be a suitable signal range for the circuitry.

4.3. SAD/SSD Operation. The cell circuitry shown in Figure 6 can provide two different output values. When $V_{\text {cont }}=\mathrm{VDD}$, switch ABS is turned off and SQ is conducting, the output of the cell will be the squared response to the input difference. If $V_{\text {cont }}=0$ and $\mathrm{ABS}$ is conducting with SQ turned off, the output current of the absolute value circuit will be directed to the cell output. This means that either the absolute value or quadratic output current can be read out from the same cell output node and either SAD or SSD operation can be selected and tested. The motion estimation process can be described as the minimization of the equation

$$
D_{N}(d x, d y)=\sum_{i=x, j=y}^{x+N_{v}, y+N_{h}}|\operatorname{ref}(i+d x, j+d y)-\operatorname{cur}(i, j)|^{p},
$$

where $N_{v} x N_{h}$ is the macroblock size, $(d x, d y)$ the applied shift vector for the reference frame, $(x, y)$ the top-left pixel of the macroblock and $p$ depends on whether SAD $(p=1)$ or SSD $(p=2)$ is applied.

The summing of the cell currents within the macroblock required for SAD/SSD is simply realized by connecting all of the output nodes of selected cells to a global output wire. Each cell receives a row and a column signal which are used to create the cell select $(\mathrm{Sel})$ signal. The cells to be summed together, that is, ones belonging to the same macroblock, are selected with peripheral row/column decoder circuitry, which is capable of addressing several rows/columns at a time in a programmable fashion. The sum currents from different macroblocks can then be evaluated, for example, with an ADC, and compared, either one block at a time in a sequential manner or with fully parallel comparison circuitry. 
The implementation of the comparison circuitry has to be carefully optimized in order to reach the best possible performance. The integration of the evaluation circuitry on the same chip as the array is a subject of further research; in the designed $32 \times 32$ test chip, the sum current is routed off-chip for measurement. However, because the SAD/SSD comparison, which is not a pixel-parallel operation, is not performed within the array itself, the cell circuitry can be kept very simple, allowing for a larger array size. Also, the measurement and evaluation circuitry can be optimized independently of the cell array, making the design more flexible and efficient.

Because the two outputs (ABS/SQ) result from different types of sources (PMOS/NMOS, resp.) also the cell selection switches $(\mathrm{Sel})$ were implemented separately for both current paths. This allowed the use of the correct type of devices, in order to minimize the effect of the switches on the output current value. The different current polarities naturally also have to be accounted for in the measurement circuitry. In the implemented chip the SAD/SSD evaluations will be performed off-chip, however, the circuitry could also be integrated in the periphery of the AME array.

\section{Implementation Issues}

The circuit operation was simulated at the transistor level with a $9 \times 9$ cell array. A $0.13 \mu \mathrm{m}$ standard CMOS technology was used with $\mathrm{VDD}=1.2 \mathrm{~V}$. A potentially difficult design issue in the proposed method is that the shifting of the reference value as a current signal makes the implementation vulnerable to resistive drops in the large number of seriesconnected switches used for the $[8,8]$ neighborhood connectivity. The resistive effects can lead to deterministic shiftdependent offsets, which may cause errors in the motion estimation process.

5.1. Resistive Effects. The analog ABS/QUAD circuitry receives as input the difference between two current-mode DAC outputs. The applied current-mode signaling may lead to resistive distance-dependent offset in the difference extraction. A large current value causes a significant resistive voltage drop over the shift switches, which are in series between the R-DAC in the source cell and the diodeconnected input transistor of the PMOS current mirror in the target cell. This lowers the output voltage of the RDAC, which leads to current variations due to channel length modulation in the DAC transistors, in the worst case the DAC transistors may even start to come out of saturation. The channel length modulation can be mitigated to some extent by using long-channel transistors in the DAC, in this case $L=4.88 \mu \mathrm{m}$ was used for the unit current source in the DACs.

In order to simplify the design, the two DACs should be of the same type (NMOS/PMOS), which means that one of the inputs has to be mirrored in order to perform the subtraction. The type of the switches used (NMOS/PMOS) is dependent on the DAC-type and on the current mirror configuration. Simulations showed that PMOS switch transistors

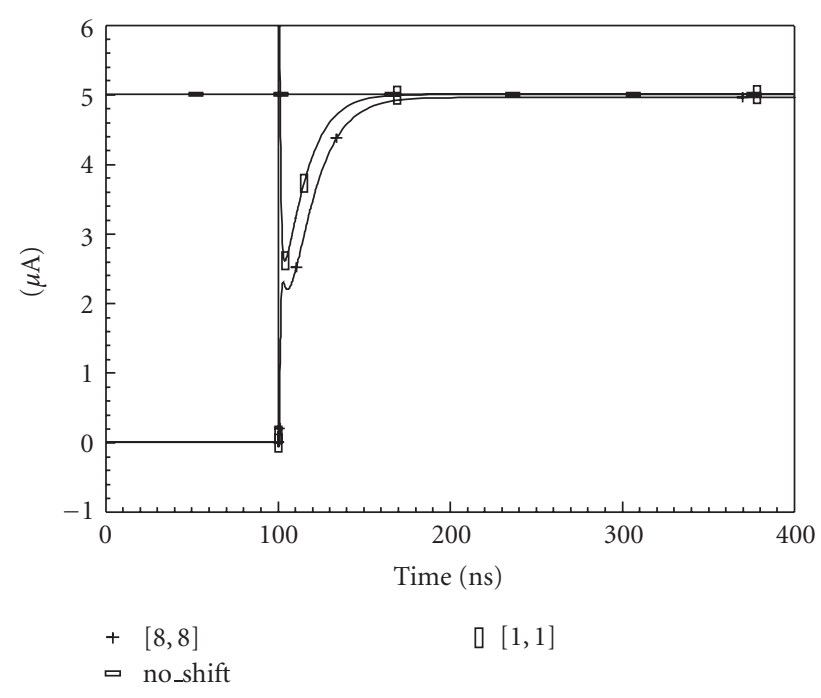

Figure 8: Transient simulations of $[0,0],[1,1]$, and $[8,8]$ shifts, with high-speed transistor switches.

of a reasonable size exhibited considerable resistive voltage drops due to their low conductance. Because this would lead to large offset errors which depend on the shift distance, NMOS transistor switches were selected. This leads to a cell configuration where the output current of an NMOStype R-DAC is propagated through (NMOS) switches and mirrored with a PMOS current mirror in the target cell as shown in Figure 5. The larger conductance of the NMOS switch transistors resulted in a much reduced distance effect, compared to PMOS switches.

The selection of the correct transistor type within the applied CMOS process can lead to considerable benefits in terms of both performance and cell area. Using lowerthreshold and higher conductivity high-speed (HS) transistors available in the CMOS technology, enables the use of smaller switches, leading to more compact cell circuitry. The larger leakage current in HS transistors is not a serious problem in this case because of the many switches in series in the shift network. Also, the voltage differences over nonconducting switches between cells in the array are relatively small, because the output current of each DAC in the array is always taken into the same resistive load (i.e., diode-connected transistor).

Figure 8 shows a transient simulation comparing three different shift operations: $[d x, d y]=[8,8],[1,1]$, and $[0,0]$ (no shift), the applied current magnitude was $5 \mu \mathrm{A}$ which is specified to be the maximum input current for the cell circuitry. The output of the R-DAC in the source cell was constant and the switch to the output neighborhood was turned on at 100 nanoseconds. These cases correspond to different numbers of switches present in the current path, that is, different resistive voltage drops. The shift network was implemented with high-speed (HS) NMOS transistors, with $W=0.5 \mu \mathrm{m}$ and $L=0.13 \mu \mathrm{m}$. The two extrema are $[0,0]$ with only 2 switches and $[8,8]$ with more than 20 switches in the current propagation path. The simulated difference in the DAC output current between theses cases 


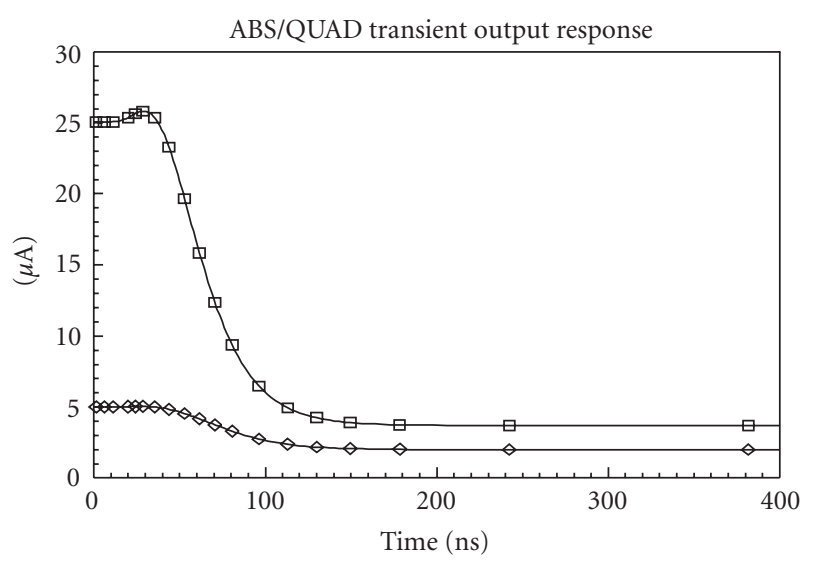

$\diamond \mathrm{ABS}$

$\square$ QUAD

FIgure 9: Transient settling of the cell output for ABS and QUAD operations.

is approximately $40 \mathrm{nA}$, with low-leakage transistors of the same size the output difference would be more than doubled.

The resistive loss in the switches should not be a limiting design issue for the proposed circuitry, since even with the maximum input value, the difference in the shifted currents is small. However, extensive simulations and testing with natural image streams on the manufactured array is necessary to verify if further optimization of the shift operation is necessary. The distance effects could be mitigated, for example, by applying cascode techniques to the DACs, to increase output resistance, or by taking the shift distance into account in the SAD/SSD evaluation phase. Other commonmode nonlinearity effects caused by the analog processing circuitry, which are not dependent on shift distance, should not be critical to the AME operation if they do not affect the ordering in the following macroblock comparison.

5.2. Mismatch Effects. Mismatch between the analog transistors in the processor cell is the most significant source of errors in the proposed motion estimation architecture. The achieved accuracy is proportional to the area of a device [13], therefore the circuitry should be made as simple as possible and, for example, unnecessary current mirroring operations should be avoided. In the shift operation, the current is only mirrored once through the local PMOS mirror. Another current mirror is required for the absolute value circuit. Because of the small number of devices, the mirror transistors can be relatively large, in the realized test chip devices with $W / L=5 / 4 \mu \mathrm{m}$ were used.

The mismatch variation in the different parts of the cell circuitry was simulated with a Monte Carlo simulator, with mismatch models provided by the manufacturer. The simulated output standard deviation of the input DAC at the full signal output of $5 \mu \mathrm{A}$ was approximately $0.5 \%$. The simulated relative output standard deviation of the absolute value current, without input mismatch, with the maximum input was approximately $0.6 \%$. The squaring operation introduces additional mismatch variation. The simulated standard deviation in the quadrature output, which also includes the absolute value variation, but not input signal mismatch, was approximately $2.3 \%$.

Fixed-pattern noise in the input image, due to the input DACs could, at least to some extent, be corrected by adjusting (predistorting) the digital input codes for different cells. The subsequent current summing operation within the whole macroblock, which results in the actual evaluated signal from the array for a given shift operation, leads to averaging of the individual cell output errors. The averaging is naturally more prominent for a larger block size. The total effect of the mismatch variation on the complete motion estimation operation is a very complex issue, since the actual realized inaccuracy is totally signal- and image-dependent. System level simulations and measurements with realistic image sizes and a real-world video streams are required to accurately characterize the performance of the AME array. This is not within the scope of this paper, but will be addressed in further research, with the help of the designed test array.

Because the analog circuitry inside each cell is very simple, the transistors can be made fairly large. However, minimal transistor area should still be targeted in order to maximize the spatial resolution or to minimize the area of the processor array. Also, because the whole image cannot be practically processed with the array at the same time, also the speed of operation should be maximized by targeting the smallest possible capacitive loads, that is, smallest possible transistors. In this respect the application of area-efficient mismatch compensation techniques in the AME cell, such as the one discussed in [14], should be considered.

5.3. Speed and Other Performance Issues. The delay in the shift operation can be observed from Figure 8 . It can be noticed that the maximum delay, in the $[8,8]$ shift, is approximately 80 nanoseconds. This delay is defined by the resistance of the shift switches and the capacitance from the current mirror in the target cell, which had a transistor size of $5 / 4 \mu \mathrm{m}$ in the final design. The settling time of the analog absolute value and squaring circuitry is shown in Figure 9, where the input difference was changed from $5 \mu \mathrm{A}$ to $2 \mu \mathrm{A}$ at time zero. It can be noticed that the outputs settle to their new values in less than 200 nanoseconds. In practical operation also the delay of the evaluation circuitry and I/O has to be considered, however, it can be seen that the switched-current cell operation is relatively fast. The effects of device mismatch on the delay are negligible.

The inclusion of in-cell DACs for providing the input frames also leads to additional benefits related to the analog circuit implementation. Because the input currents are always provided by active DACs and no dynamic current memories are used, effects such as charge injection and memory retention problems due to leakage are not an issue, since the input values are static and robust. An important issue to be considered in further research, in terms of performance, is the optimal implementation of the array I/O and especially the writing of the in-cell DACs, so that a large image frame can be processed fast enough and with sufficiently low power consumption. 


\section{AME Test Array}

A $32 \times 32$ cell test array was designed in the $0.13 \mu \mathrm{m} 6 \mathrm{M}$ digital CMOS technology with the high-speed transistor option, for evaluating the performance of the proposed motion estimation approach in practice. The chip enables the evaluation of the motion estimation operations within a $[8,8]$ search range and a $16 \times 16$ maximum block size. The size of the actual active array is $16 \times 16$ cells, an 8 -cell wide boundary is required for providing input values to the $16 \times 16$ cell active area, with a maximum shift distance of 8 cells. In practice the analog processing circuitry in the boundary cells is unnecessary, however, in the test chip layout design it was simpler to just use basically the same cell for the boundary, although with some wires and controls disconnected. Also, because the DACs (+memories) and the shift network take up most of the cell area, as can be seen from Figure 11, leaving out the processing circuitry would not have lead to significant area savings.

The layout of the array is shown in Figure 10. The layout of a single array cell is shown in Figure 11, with the different functional sections of the cell highlighted. The size of the chip is approximately $1.5 \times 1.7 \mathrm{~mm}^{2}$ and the size of a single cell is approximately $30 \times 35 \mu \mathrm{m}^{2}$. The periphery of the array includes row-wise buffers for the global control signals and address decoders which enable the simultaneous selection of multiple rows/columns for different block-size summation operations. The sum current to be evaluated is available from a global wire, which is only connected to the outputs of the active $16 \times 16$ cell array and taken to a chip output pad for external evaluation.

The digital control for the array as well as the evaluation and comparison of the SAD/SSD results will be initially realized with an additional FPGA chip and an off-chip ADC. For a complete motion estimation processor realization also these operations have to be optimized and implemented with dedicated on-chip circuitry for optimal performance. However, at this stage a more thorough examination of the analog cell array through chip measurements is required to validate the feasibility of the approach, for example, in terms of speed and accuracy, and to derive more exact specifications for the remaining hardware and the whole system. Many design choices, such as the practical array size are still open to optimization.

6.1. Related Work. Since the complete motion estimation system has not been realized at this time, it is difficult to directly compare the performance of the proposed circuitry to other implementations. Also, the total system performance is a compromise between multiple factors, such as picture quality, bitrate, power consumption, and cost (i.e., silicon area). The performance of the underlying analog processing hardware has to be first evaluated in detail with measurements before making quantitative comparisons to other implementations. For example, the practical accuracy and robustness of the proposed analog processing, which is difficult to examine comprehensively with simulations, also affects the choice of the ME algorithm. The choice between implementing a full search operation and for example, a

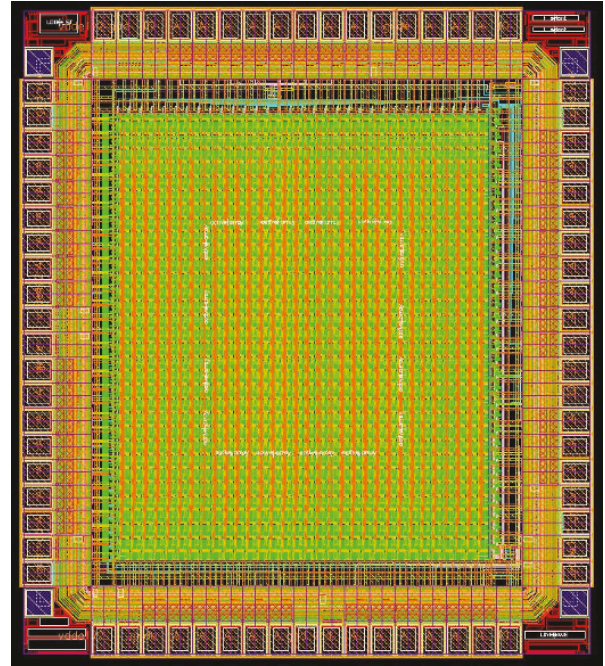

FIgURE 10: Layout of the $32 \times 32$ AME processor array. The active processing area of $16 \times 16$ cells is highlighted in the middle or the array.

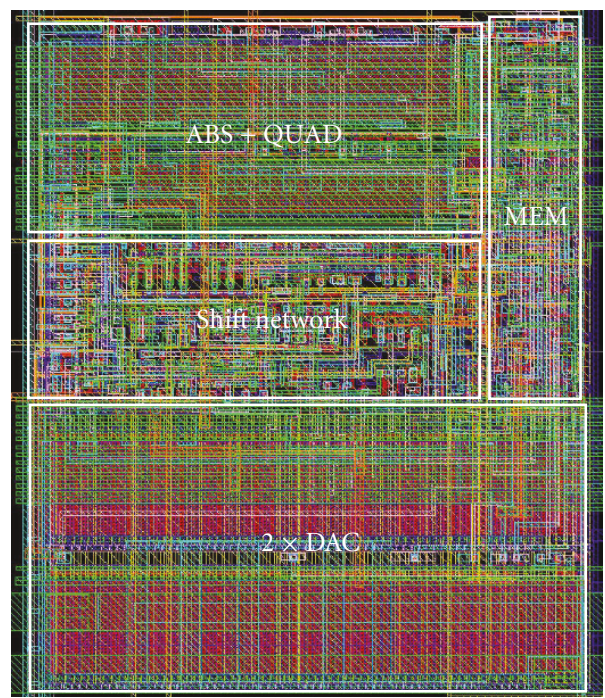

FIGURE 11: Layout of a single array cell with different functional sections highlighted.

gradient descent search (GDS) algorithm, has a very large effect on the number of required pixel operations, that is, also on the power consumption. However, the parameters reported from ME implementations in the field are briefly reviewed to estimate the performance that should be targeted and improved upon.

Few separate ME realizations have been presented. Current ME realizations are typically embedded within full audio-video codecs. Comparing the presented work to such implementations is difficult due to the fact that little specific information (such as power consumption) on the ME part is available. The most comprehensively reported motion estimation implementations have been digital chips. In [15], a $0.4 \mathrm{~mW}$ (QCIF@15 fps, $0.85 \mathrm{MHz}$ )/2.5 mW 
(CIF@30 fps, $6.75 \mathrm{MHz}$ ) motion estimation IC, in $0.18 \mu \mathrm{m}$ technology with a $1.0 \mathrm{~V}$ power supply, using a gradient descent search algorithm was presented. As the design does not incorporate frame memory the stated power consumption figures do not include the data transfer between the frame memory and local search area memories. In [15], it is also estimated that the power consumption of a Full Search QCIF@15 fps ME IC would be in the range of $20 \mathrm{~mW}$. In [16], a 16.2 mW QCIF@15 fps motion estimation IC using pixel wordlength truncation was presented. The chip has a $20 \mathrm{MHz}$ clock frequency (the operating voltage was not stated) and is implemented with $0.18 \mu \mathrm{m}$ technology. The design incorporates frame memories whose portion of the power consumption is $11.7 \mathrm{~mW}$. In [17], a $1920 \times 1080$ HDTV@30 fps ME core was presented. The ME core is designed for a power supply of $1.0 \mathrm{~V}$ and a clock frequency of $81 \mathrm{MHz}$ and is implemented with $0.13 \mu \mathrm{m}$ technology. The estimated power consumption is $65 \mathrm{~mW}$ without frame memories.

For H.264 [18] presents a 720×480 SVGA@30 fps systolic array design that incorporates Full Search for the seven different block sizes of H.264. With $0.35 \mu \mathrm{m}$ technology and a clock frequency of $67 \mathrm{MHz}$ (the operating voltage is not stated) the design has a simulated power consumption of $737 \mathrm{~mW}$ without frame memories. In [19], a QCIF@15 fps implementation using variable block-size Full Search is presented. With $0.13 \mu \mathrm{m}$ technology, a clock frequency of $6.7 \mathrm{MHz}$, and an operating voltage of $1.2 \mathrm{~V}$ the design has a simulated power consumption of $9.1 \mathrm{~mW}$ without frame memories. Both of these variable block-size ME implementations operate by computing the distortion measure values for the smallest block size and then combining these values to form the distortion measures for the larger block sizes. Also, neither of these designs comments on the choice of the optimal block size.

In other proposed block-based analog motion estimation approaches [2, 3], although the computation, memory, and data transfer are analog, the architectures resemble conventional digital ME processors. This is in contrast to the proposed work which proposes an interconnected analog parallel processor architecture. In both $[2,3]$, only the picture quality results are presented which, without presenting the effect on bit-rate, is not fully meaningful and makes comparison to other implementations difficult. For the CNN-based image stabilization architecture presented in [4] no power consumption or processing figures were given. Additionally, the effect of error in the ME distortion measure has been studied in [20,21].

At the time of writing the designed $32 \times 32$ cell chip is being manufactured and measurement results, further analysis and comparison to the other implementations, based on experimental results, will be presented in future publications.

\section{Conclusion}

This paper presented an analog motion estimation array with a new cell neighborhood configuration and the required circuitry for reference image shifting. In the otherwise very simple AME cell architecture, the shift network is clearly the most complex aspect of the implementation. Compared to a previously proposed method, considerable savings in array level interconnect complexity are achieved. The new shift method thus allows for a more efficient implementation of the analog motion estimation array. Transistor level simulations show that the method and the related analog processing circuitry can be applied for high-speed operation and with sufficient accuracy. A realistic performance comparison with other proposed motion estimation circuit architectures can be achieved in future work, based on measurements of the implemented $32 \times 32$ test array.

\section{Acknowledgment}

This work has been supported by the Academy of Finland projects 107645 and 123354 .

\section{References}

[1] L. O. Chua and L. Yang, "Cellular neural networks: theory," IEEE transactions on Circuits and Systems, vol. 35, no. 10, pp. 1257-1272, 1988.

[2] A. Tomasini, M. Brattoli, E. Chioffi, G. Colli, D. Gerna, and M. Pasotti, "B/W adaptive image grabber with analog motion vector estimator at 0.3 GOPS," in Proceedings of the 42nd IEEE International Solid-State Circuits Conference (ISSCC '96), pp. 94-95, San Francisco, Calif, USA, February 1996.

[3] M. Panovic and A. Demosthenous, "A low-power analog motion estimation processor for digital video coding," IEEE Journal of Solid-State Circuits, vol. 41, no. 3, pp. 673-683, 2006.

[4] Y.-C. Cheng, J.-F. Chung, C.-T. Lin, and S.-C. Hsu, "Local motion estimation based on cellular neural network technology for image stabilization processing," in Proceedings of the 9th IEEE International Workshop on Cellular Neural Networks and Their Applications (CNNA '05), pp. 286-289, Hsinchu, Tawian, May 2005.

[5] L. Koskinen, J. Marku, A. Paasio, and K. Halonen, "Architecture for analog variable block-size motion estimation," in Proceedings of the 14th IEEE International Conference on Image Processing (ICIP '07), vol. 2, pp. 493-496, San Antonio, Tex, USA, September 2007.

[6] L. Koskinen, K. Halonen, and A. Paasio, "Efficient shift of reference data in analog motion estimation," in Proceedings of the 9th IEEE International Workshop on Cellular Neural Networks and Their Applications (CNNA '05), pp. 130-133, Hsinchu, Tawian, May 2005.

[7] L. Koskinen, A. Paasio, and K. Halonen, "3-neighborhood motion estimation in CNN silicon architectures," in Proceedings of IEEE International Symposium on Circuits and Systems (ISCAS '04), vol. 5, pp. 708-711, Vancouver, Canada, May 2004.

[8] J. Marku, L. Koskinen, and A. Paasio, "A 130 nm implementation of analog variable block-size motion estimation cell," in Proceedings of the International Symposium on Integrated Circuits (ISIC '07), pp. 57-60, Singapore, September 2007.

[9] J. Poikonen, M. Laiho, A. Paasio, L. Koskinen, and K. Halonen, "Interconnect-efficient reference data shift for optimized analog motion estimation," in Proceedings of the 11th IEEE International Workshop on Cellular Neural Networks and Their Applications (CNNA '08), pp. 102-107, Santiago de Compostela, Spain, July 2008. 
[10] A. Rodríguez-Vázquez, R. Domínguez-Castro, F. Medeiro, and M. Delgado-Restituto, "High resolution CMOS current comparators: design and applications to current-mode function generation," Analog Integrated Circuits and Signal Processing, vol. 7, no. 2, pp. 149-165, 1995.

[11] J. Poikonen and A. Paasio, "An area-efficient full-wave current rectifier for analog array processing," in Proceedings of IEEE International Symposium on Circuits and Systems (ISCAS '03), vol. 5, pp. 757-760, Bangkok, Thailand, May 2003.

[12] L. Koskinen, M. Laiho, A. Paasio, and K. Halonen, "Motion estimation matching criterion computation with analog circuits," in Proceedings of the 8th International Workshop on Cellular Neural Networks and Their Applications (CNNA '04), pp. 216-221, Budapest, Hungary, July 2004.

[13] M. J. M. Pelgrom, A. C. J. Duinmaijer, and A. P. G. Welbers, "Matching properties of MOS transistors," IEEE Journal of Solid-State Circuits, vol. 24, no. 5, pp. 1433-1439, 1989.

[14] J. Marku, K. Virtanen, J. Maunu, J. Poikonen, and A. Paasio, "Current mismatch and nonlinearity compensation in mixedmode array processors," in Proceedings of the 11th IEEE International Workshop on Cellular Neural Networks and Their Applications (CNNA '08), pp. 75-80, Santiago de Compostela, Spain, July 2008.

[15] M. Miyama, J. Miyakoshi, Y. Kuroda, K. Imamura, H. Hashimoto, and M. Yoshimoto, "A sub-mW MPEG-4 motion estimation processor core for mobile video application," IEEE Journal of Solid-State Circuits, vol. 39, no. 9, pp. 1562-1570, 2004.

[16] C.-W. Yoon and H.-J. Yoo, "Low power motion estimation and motion compensation block IPs in MPEG-4 video codec hardware for portable applications," IEICE Transactions on Electronics, vol. E86-C, no. 4, pp. 553-560, 2003.

[17] M. Miyama, O. Tooyama, N. Takamatsu, et al., "An ultra low power motion estimation processor for MEPG2 HDTV resolution video," IEICE Transactions on Electronics, vol. E86C, no. 4, pp. 561-569, 2003.

[18] Y.-W. Huang, T.-C. Wang, B.-Y. Hsieh, and L.-G. Chen, "Hardware architecture design for variable block size motion estimation in MPEG-4 AVC/JVT/ITU-T H.264," in Proceedings of IEEE International Symposium on Circuits and Systems (ISCAS '03), vol. 2, pp. 796-799, Bangkok, Thailand, May 2003.

[19] S. Y. Yap and J. V. Mccanny, "A VLSI architecture for advanced video coding motion estimation," in Proceedings of the 14th IEEE International Conference on Application-Specific Systems, Architectures, and Processors (ASAP '03), pp. 293-301, The Hague, The Netherlands, June 2003.

[20] M. Tartagni, A. Leone, A. Pirani, and R. Guerrieri, "A blockmatching module for video compression," in Proceedings of IEEE Symposium on Low Power Electronics, pp. 24-25, San Diego, Calif, USA, October 1994.

[21] M. Panovic and A. Demosthenous, "Architectures for analog motion estimation processors: a comparison," in Proceedings of IEEE International Symposium on Circuits and Systems (ISCAS '05), vol. 5, pp. 4566-4569, Kobe, Japan, May 2005. 